\section{Measurement of}

Gold Ballbond

Intermetallic

Coverage

\author{
F Wulff ${ }^{1}$, CD Breach ${ }^{2}$ \\ ${ }^{1}$ Materials \& Applications Centre, Kulicke and Soffa \\ (S.E.A.) Pte. Ltd., \#04-05 TECHplace II, Block 5002, \\ Ang Mo Kio Avenue 5, Singapore 569871 \\ ${ }^{2} 166$ Lentor Loop, Bullion Park Tower 3, Singapore \\ 789097 \\ ${ }^{1}$ Corresponding author: \\ E-mail: fwulff@kns.com
}

\begin{abstract}
An important characteristic of gold ballbonds is intermetallic coverage (IMC), defined as the percentage of the total area on which gold aluminide compounds are formed by reaction between the gold ball and aluminium alloy metallization during thermosonic bonding. IMC measurement complements mechanical measurements such as ball pull and shear strength and in general, IMC should be maximised to aid in achieving a high degree of initial mechanical robustness so that devices can withstand subsequent processing steps and maintain high reliability. Too little IMC can result in weak bonds and erratic intermetallic growth patterns during subsequent thermal processing of bonded devices and may result in mechanical and electrical malfunction and failure. Optical microscopy of the underside of ballbonds is often used to determine IMC, but usually without consideration of certain key physical effects such as lighting, resolution and surface roughness. This paper compares the relative merits of IMC measurement by scanning electron microscopy (SEM) and optical microscopy and includes practical guidelines for the use of each technique.
\end{abstract}

\section{Keywords}

gold ballbond, intermetallic coverage, microscopy

\section{Introduction}

Welding between gold balls and aluminium alloy metallization during thermosonic bonding occurs by solid state intermixing of $\mathrm{Au}$ and $\mathrm{Al}$ at the interface and formation of gold-aluminium intermetallic phase. The proportion of the total bond area that consists of this intermetallic phase is commonly referred to as intermetallic coverage, abbreviated to IMC. Ultrasonics is essential in forming IMC by means of friction [1-3] but is not uniform across the interface and starts as discrete islands that grow under the action of ultrasonics and eventually anchor the ball to the Al metallization. If bonding parameters are optimised, most of the interfacial area, as much as $70-80 \%$, should consist of IMC. The minimum amount of IMC needed for the Au-AI interface to be mechanically robust during pull testing need only be a little larger than the cross-sectional area of the wire. However, having large areas of the interface unbonded can permit air, airborne contaminants and epoxy moulding compound to penetrate underneath the ball, leading to potential oxidation and corrosion reactions during subsequent assembly steps. Maximizing IMC is therefore an essential part of optimizing the ballbonding process.

Measurement of IMC is normally performed by dissolving away the Al bondpad with $\mathrm{KOH}$ solution that does not attack intermetallics or gold [4] and viewing the underside of the ball. Determining the precise amount of $I M C$ required to make a robust ballbond is not an exact science, but an empirical guideline is that $70 \%$ of the true bonded ball area should consist of Au-Al intermetallic compounds. There are two common methods used to view and record the intermetallic coverage from an image of the underside of a gold ball for subsequent measurement using image analysis software. The first is by using light microscopy (LM) and the second by scanning electron microscopy (SEM). SEM requires specimens to be gold coated, and placed in the SEM chamber, then pumped down and examined while LM, which doesn't require special and time consuming specimen preparation, is perceived to be faster and easier than SEM. However, each method has its merits and there are certain factors, especially with LM, which need to be understood to correctly measure IMC. Different illumination modes can be used for light microscopy and unlike SEM sample alignment under the microscope and lighting may complicate the identification and measurement of IMC and can easily lead to erroneous measurements.

In assembly engineering qualifications of semiconductor packages, optical assessment of the intermetallic coverage appears to be preferred because it is less time consuming. However, while optical assessment of coverage is faster, it is also more difficult to interpret. During the assembly engineering phase of a new package qualification it may be desirable to have the detail afforded by SEM measurements of IMC. However, during mass production optical measurements may be more appropriate because they are less time consuming. The aim of this paper is to provide an 
objective comparison of optical and SEM measurement of intermetallic coverage, highlighting the advantages and disadvantages of each technique and recommending best practices.

\section{Experimental}

$4 \mathrm{~N}$ (99.99\% purity) wires of $18-25 \mu \mathrm{m}$ diameter were bonded to on $10,000 \AA$ thick $\mathrm{Al}-0.5 \% \mathrm{Cu}-1 \% \mathrm{Si}$ metallization spaced 45-60um apart on Si chips. The chips were mounted onto plastic ball grid array (BGA) substrates. Prior to bonding, devices were plasma cleaned with Ar. Bond site temperature was set at $170^{\circ} \mathrm{C}$. Optimum ballbond (1st bond) parameters were obtained using response surface methodology with free air ball (FAB) size, contact velocity (CV), power, time and force as the variables and a population size of 30 balls for each type of device. Statistical software was used to analyse the data and optimum parameters were confirmed by bonding 60 balls and measuring the shear and pull values using a Dage 4000 series wirebond pull/shear tester.

Bonded balls were removed by dissolving the Al alloy bondpad using $\mathrm{KOH}$ etching in which $3 \mathrm{~g} \mathrm{KOH}$ pellets are firstly dissolved in $100 \mathrm{ml}$ water and heated up to $70^{\circ} \mathrm{C}$ (the $\mathrm{KOH}$ concentration can be raised if a higher etching rate is required). The lead frame or BGA is placed into the solution for 20 minutes and then rinsed with DI water. Balls easily separate from the bondpad and the wire can be manually bent to orient the underside of the ball for viewing. Light microscopy (LM) was performed with a Leica DMRM microscope. A Leo 1450 VP SEM was used for secondary electron imaging.

\section{Results and discussion}

\subsection{Appearance and identification of coverage by SEM and light microscopy}

An example of the same ball viewed with SEM, bright field optical microscopy and dark field optical microscopy without image processing is shown in Fig. 1. The scale of the images is typical for making measurements on ballbonds, with the image filling as much of the frame as possible while allowing the complete outline of the ball to be viewed. It is very important that the image is perpendicular to the viewing direction, more so for optical microscopy which has poorer depth of field than SEM. The SEM image (Fig. 1(a)) clearly shows IMC as light grey features, slightly raised, with the noncovered (non-bonded) regions clearly visible as dark grey. However, the excellent depth of field of SEM also makes identification of the true contact area more difficult. A bright field image of the same ball in Fig 1(b) shows IMC as dark grey while the non-bonded regions are clearly visible as bright areas (yellow in colour). There is little doubt about the true contact area because light from the non-planar regions is not reflected back to the objective lens. However, whereas
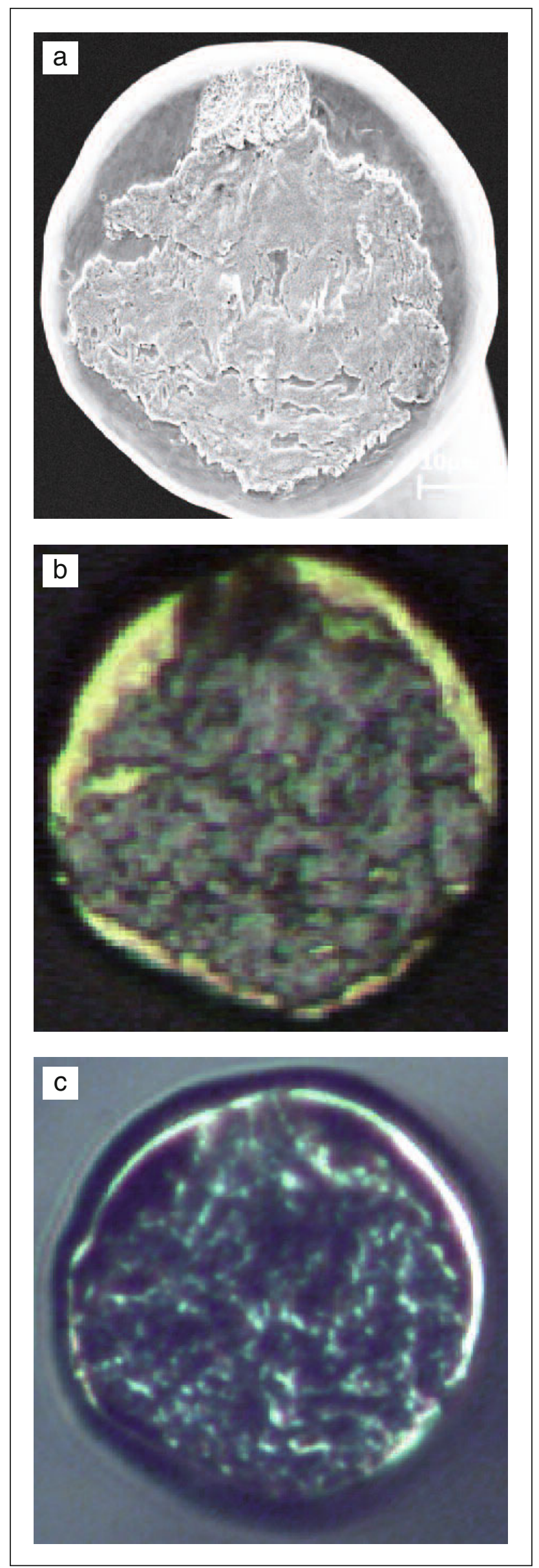

Figure 1

Underside of the same ball viewed (a) by SEM (b) by bright field optical microscopy (c) dark-field optical microscopy 
the non-covered regions are distinct in SEM (Fig. 1(a)), it is difficult to determine if the black lines in the optical image in Fig. 1(b) are non-bonded regions or boundaries between non-bonded areas and IMC. The regions indicated in Fig. 1(a) and Fig. 1(b) illustrate the difficulty of identifying whether the region is coverage and the size of that region. Surface roughness also affects the image interpretation because rough surfaces tend to scatter more light away from the objective lens making it difficult to know if the dark regions are intermetallic coverage or non-bonded regions, as emphasised in area B of Fig. 1. Another feature of the optical measurements is the inability to distinguish ball deformation from IMC such as area D in Fig. 1(a), which when examined at higher magnification as in Fig. 2 is seen to be a rough surface due to ball deformation. With bright field LM the same region appears dark and with dark field microscopy, in Fig 1(c), the same features that are bright in reflected light microscopy become dark (see Appendix for a brief description of optical microscopy and $[5,6]$ for more details).

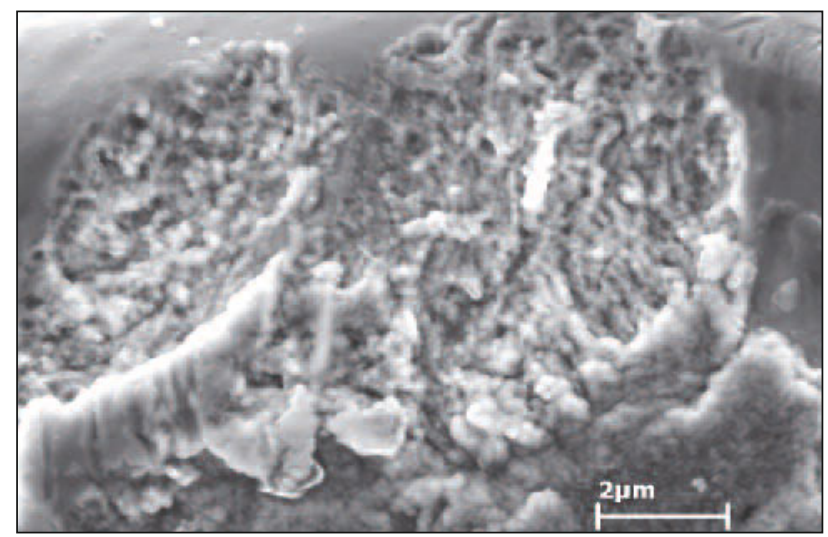

Figure 2

High magnification SEM image of region A in Fig. 1(a) showing rough area of ball

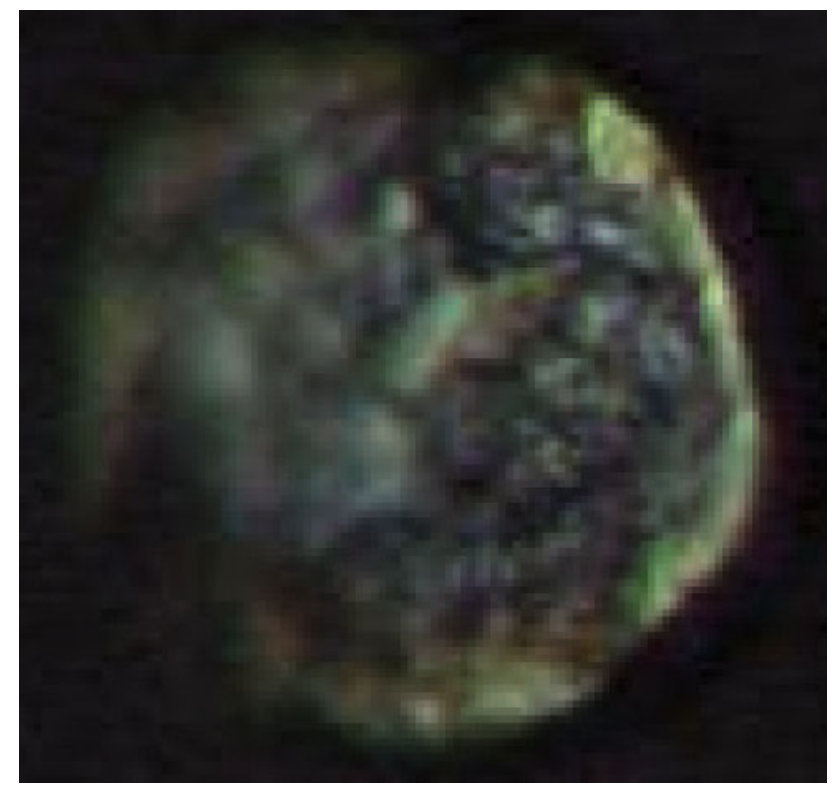

Figure 3

Optical image of coverage illustrating the poor depth of field and difficulty of alignment and focusing with optical microscopy
An important factor is planarity of the ball relative to the objective lens. This is especially important in optical microscopy. There is a perception that optical microscopy is easy and quick for assessing IMC. However, aligning twenty balls under an optical microscope one at a time so they are perpendicular to the objective lens can take as much or more time than viewing and taking images of the same number of balls in an SEM, depending partly on operator skill. Achieving ball planarity is difficult and the optical microscope is not forgiving of misalignment, as Fig. 3 illustrates. With such misalignment, the true contact area is difficult to distinguish and the perceived advantage of LM (speed) is immediately lost. An SEM on the other hand allows relatively quick and easy alignment of the ball and has good depth of field.

Another very important feature of optical microscopes that affects the ability to resolve and distinguish features is frame size. The objective lens is limited in resolution and therefore digitising images can contribute to poor resolution. An example is given in Fig. 4 using a standard camera with a resolution of $1000 \times 1000$ pixels. The captured area of the image is much larger than the ball, which is restricted already in its magnification, thus the resolution of the optical lens should be compared with those of the camera shows that the magnified image has a pixel resolution of $\sim 200 \times 200$.
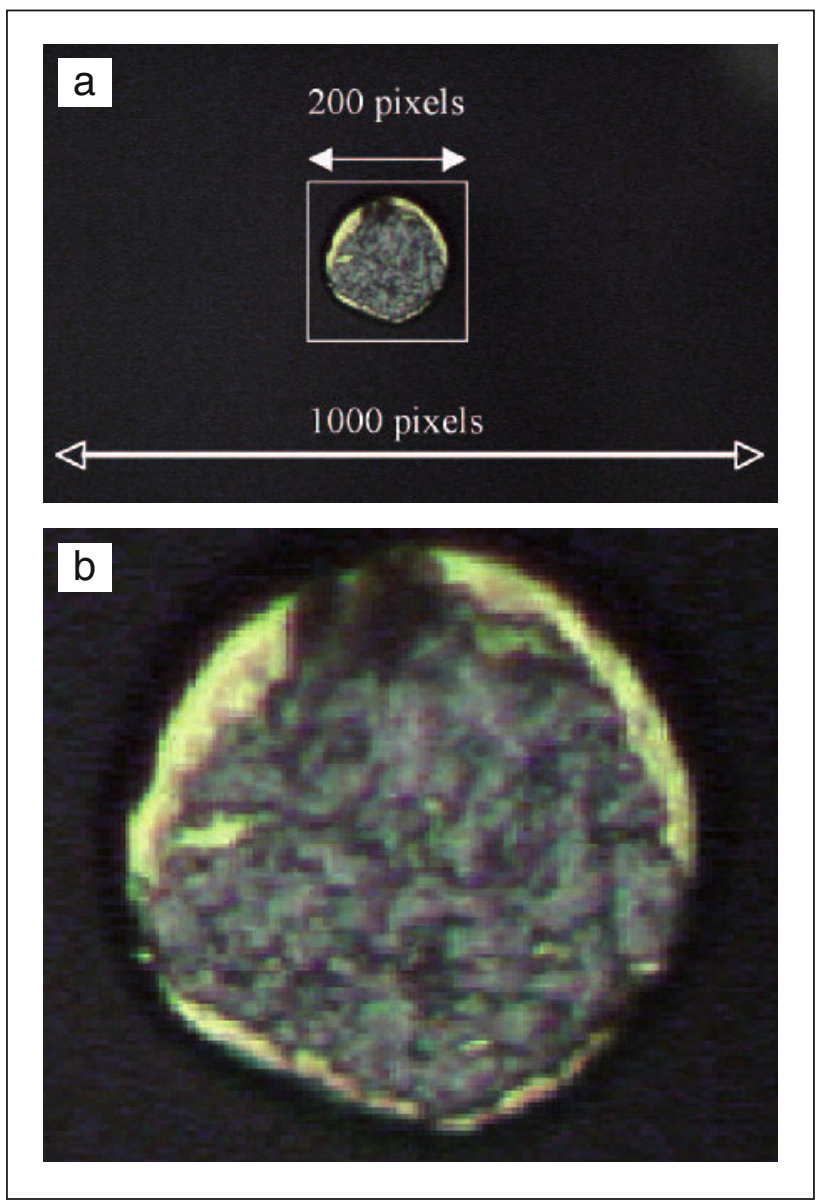

Figure 4

(a) Digital image captured with large frame size digital camera of $1000 \times 1000$ pixels (b) same image as in (a) but cropped and enlarged with software. The image is pixelated and resolution for the ball is now $\sim 200$ pixels 


\subsection{Other illumination modes in optical microscopy}

The use of monochromatic light via the use of filters can offer advantages in resolution, although, whether the human eye can resolve the difference is another matter. However, the use of green light for example is beneficial for the operator making many measurements because it reduces eye fatigue [5]. Usually objective lenses with long working distances are used in bonding application laboratories because they allow greater clearance between the objective lens and sample (see Appendix for further explanation). The effect of different lighting on the appearance of coverage for the same specimen is shown in Fig. 5. Once again, BF (Fig. 5(a)) and DF (Fig. 5(b)) images are presented for reference together with the effect of a green filter (Fig. 5(c)) and a blue filter (Fig. $5(d))$. Interpretation of these images is made easier by the ball surface being perpendicular to the axis of the objective lens. The BF image in Fig. 5(a) shows the intermetallic coverage is dark yellow and the non-bonded regions gold coloured. The DF image shows the opposite contrast. The ability to see the coverage pattern in Fig. 5(c) however is impaired by the use of green light compared to the BF image, while the use of blue light in Fig. 5(d) clearly offers no advantage over the BF imaging.

\subsection{Measurement of coverage}

Assessing the true IMC 'at a glance' from images such as those in Fig. 1 is clearly not possible, although such 'guesstimates' of IMC are not uncommon, even with images as poor as Fig. 3. A quantitative measurement of coverage is therefore required and a method used for SEM imaging that applies equally to optical imaging is now described.

The steps in the measurement of intermetallic coverage are shown in Fig. 5 The underside of ball is first marked into the contact area (circled in black) and the non-contact area that is the outside of the ball. The contact area is then cut out. These steps can be performed with any commercial image processing software but it is important that highresolution image files like TIFF be used because later image processing steps require adjustment of the pixel greyscale, and insufficient pixels will make the analysis more difficult. The image is then masked so that coverage is separated from the unbonded regions of the ball. This operation requires that each island of intermetallic be selected. The intermetallic and the background are then converted to greyscale images and the total pixel counts used to calculate the percentage of coverage. It is important that the greyscale image match the coverage in the original SEM image. The images Fig 6 are relatively easy to mask and
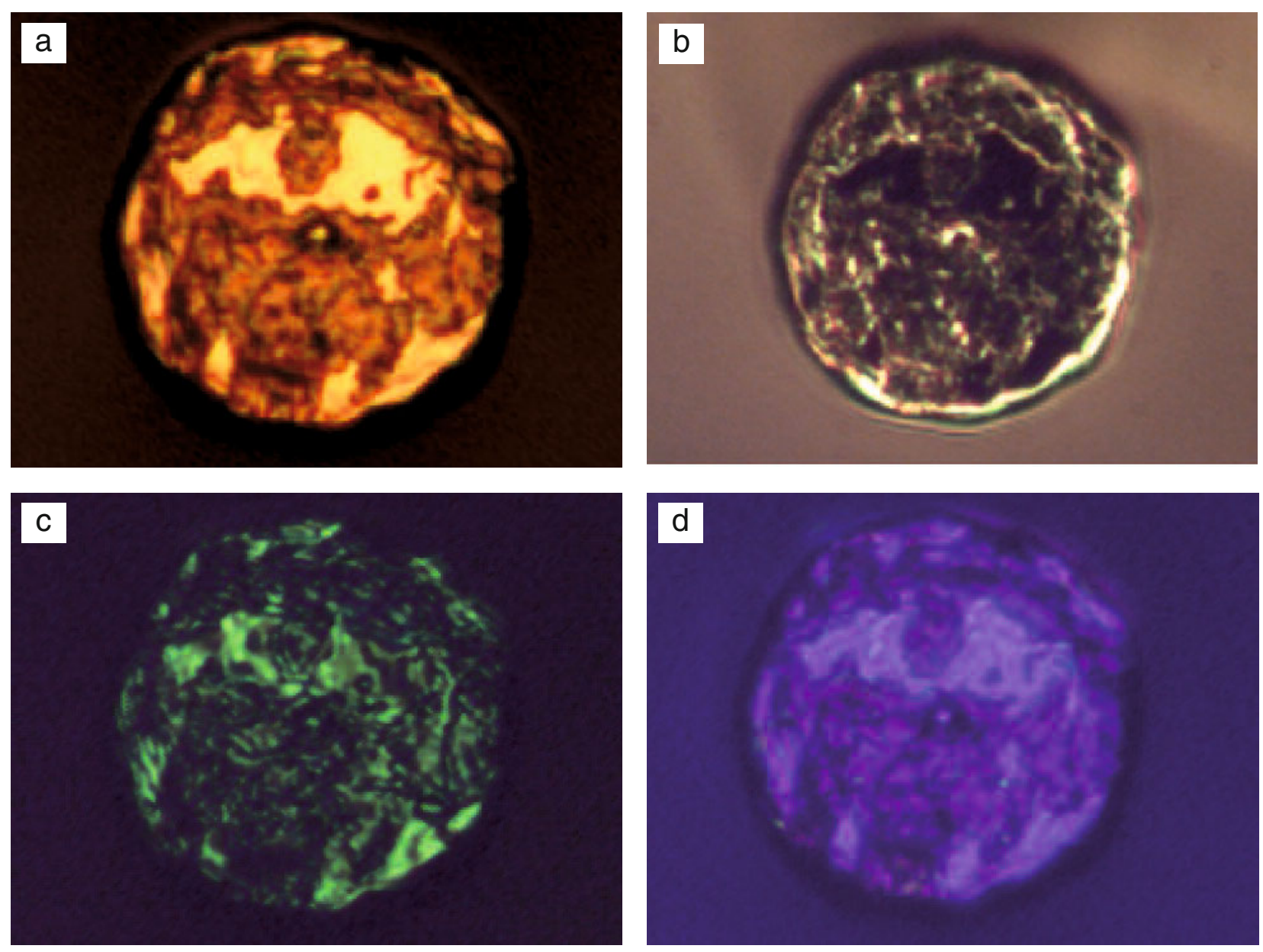

Figure 5

A series of optical images of the same ball taken with (a) BF (b) DF (c) green light (d) blue light 

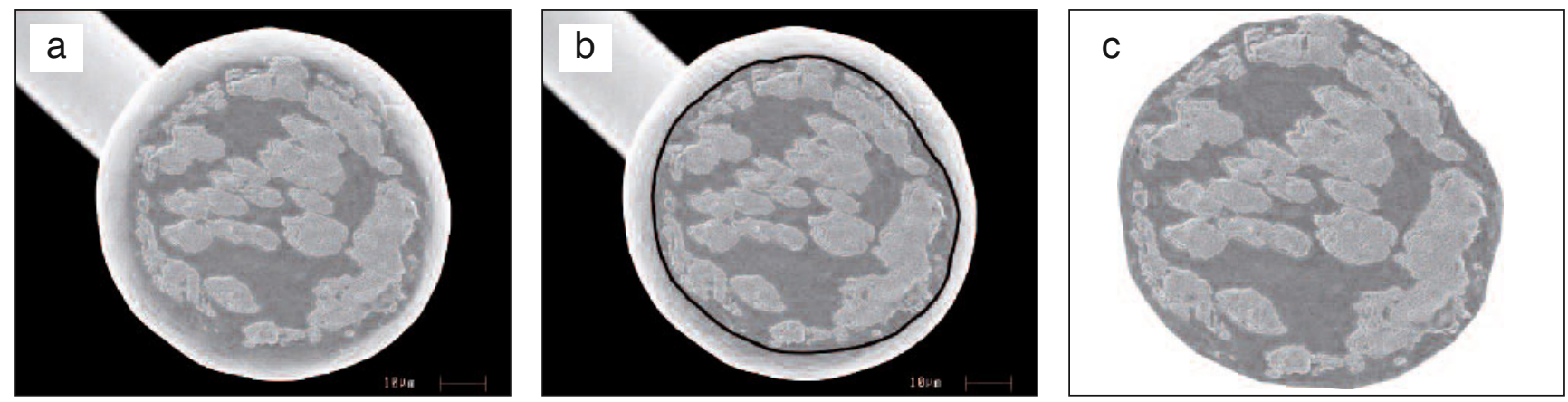

\section{Figure 6}

Sequence of SEM images illustrating the procedure for measuring intermetallic coverage by SEM. (a) ball removed from bondpad (b) marking of contact area (c) removal of noncontact region of ball leaving only area to be measured (d) digitised SEM image with nonbonded regions masked as black (e) digitised SEM with coverage masked as grey
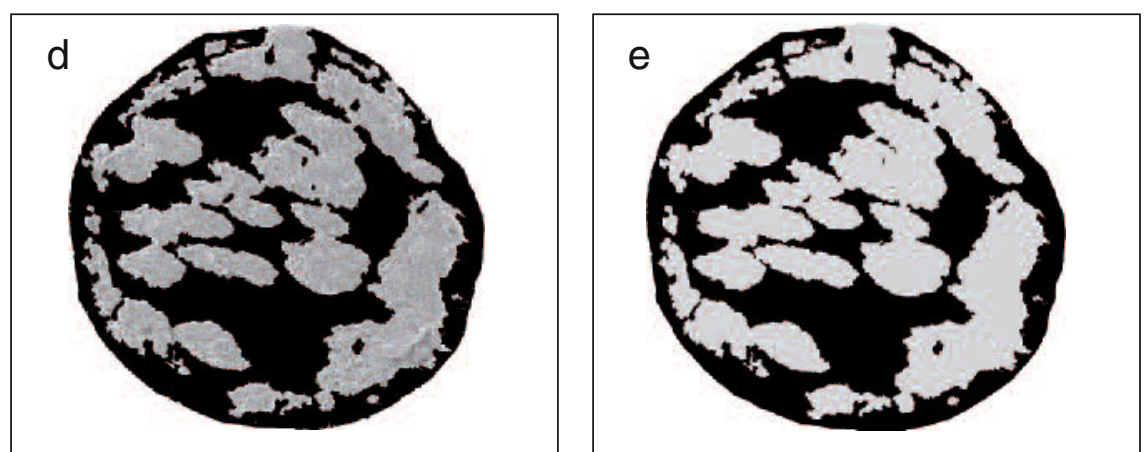

convert such that the greyscale image matches the SEM image. The same procedure can be performed with optical images, in which case the identification of the contact area is easier only if the sample is planar to the viewing direction. It cannot be emphasised strongly enough that planarity is essential for measuring intermetallic coverage using optical imaging. Using images such as Fig. 3 to assess coverage will introduce greater uncertainty in IMC measurement.

As shown earlier, coverage identification is not as straightforward with optical imaging compared with SEM, and in general, there is a difference in percentage coverage estimates with each method. This is illustrated by measuring the coverage of the ball of Fig. 1, for which the digitised images are shown in Fig. 7. The digitised images show how regions of non-coverage that are clearly visible in SEM can be mistaken for coverage when using optical microscopy. In general, optical imaging estimates larger coverage than SEM imaging because it can be more difficult to distinguish coverage from surface roughness (see section 3.2). The difference, as shown in Fig. 8, can be up to $20 \%$ larger with

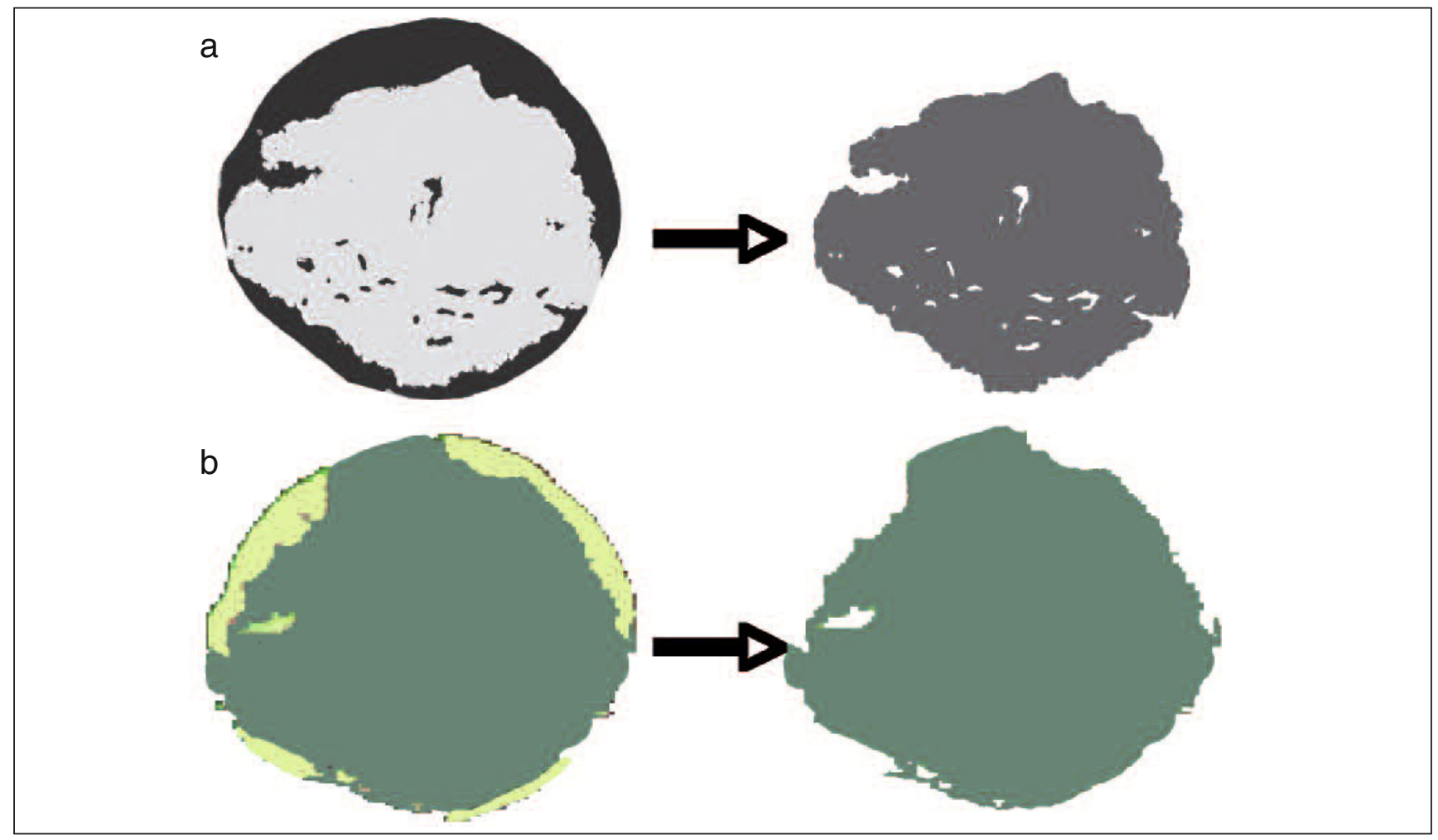

Figure 7

Digitised images for the ball in Fig. 2. Left images show contact area and right images show coverage. (a) SEM image (b) optical image 


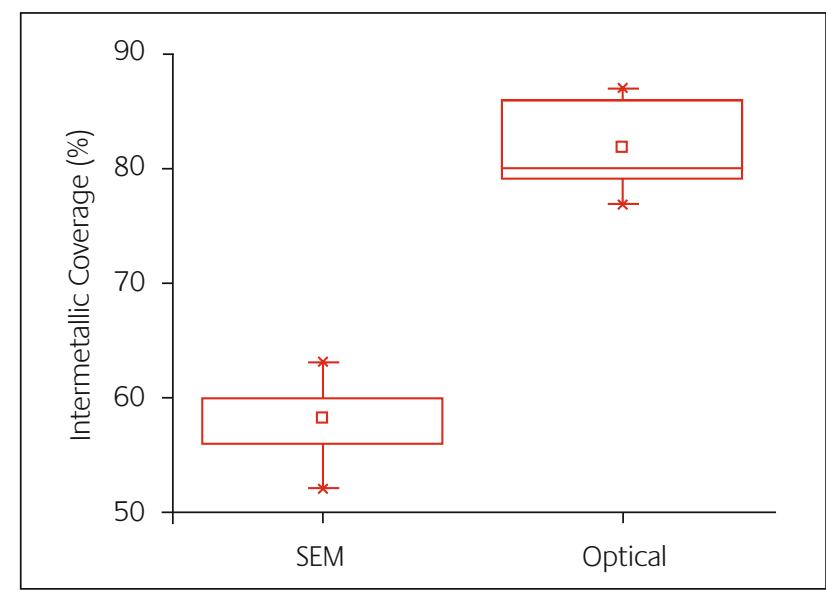

Figure 8

Comparison of intermetallic coverage measured using SEM and BF optical microscopy

measurements made from optical images. In general, SEM is recommended for engineering qualifications, at least during the first pass selection of wire materials, when it is a good idea to take SEM and optical images of the same samples. Comparison of good and bad coverage images and the correlation between the SEM and optical images can be used as an aid for mass production operations in process control, where SEM would definitely be very time consuming and impractical. Correct assessment of IMC at the early stages of
Table 1

Recommended average coverage by pitch

Application

Average Coverage

\begin{tabular}{ll}
\hline Fine Pitch & $\geq 70 \%$ \\
\hline Ultra Fine Pitch & $\geq 60 \%$ \\
\hline
\end{tabular}

package qualification, while possibly involving a little more effort, can save time and effort when problems occur during later stages of qualification.

\subsection{Sample contamination effects on coverage measurement}

The amount of information contained in optical images is strongly dependent on the surface roughness that can scatter light away from the objective lens and that there should be no doubt about the ability of surface features on the underside of a ball, such as roughness or specks of dirt, to scatter light and give a completely unrepresentative optical image. An example of how optical imaging alone can be misleading about the degree of IMC is shown in Fig. 9, which shows an optical and SEM image of the same ball. The optical image and the coverage measurement do not at all give a true idea of how contaminated the underside of the ball really is, and without the SEM image, the coverage calculation would be in serious error.

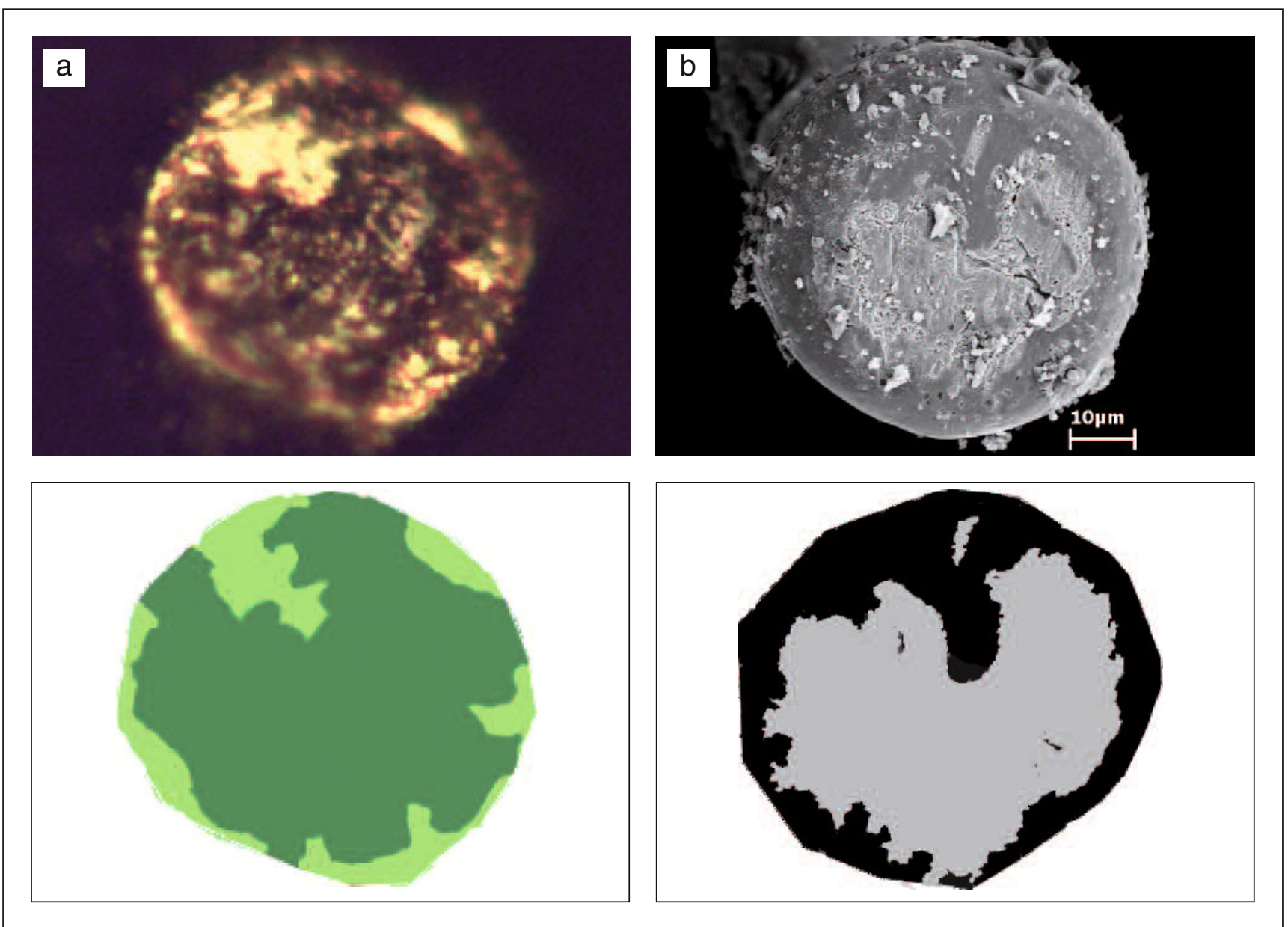

Figure 9

Effect of contamination on appearance of coverage and coverage patterns (a) optical image (top) and digitised coverage pattern (b) SEM image (top) and digitised coverage pattern 

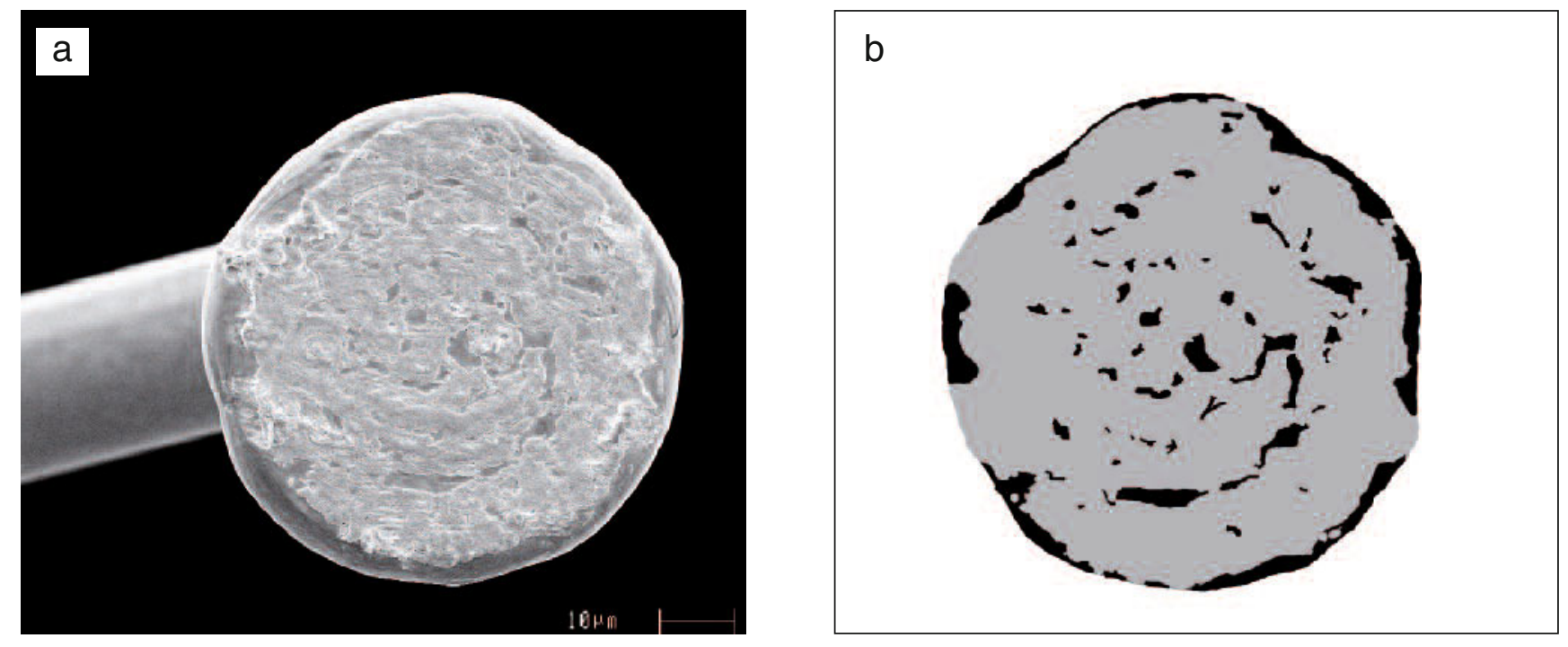

Figure 10

(a) SEM, SE image of the underside of a typical gold ball after removal from a bondpad. Light areas are regions of intermetallic. (b) Corresponding binary image with $84 \%$ calculated coverage, showing unbonded regions as dark areas

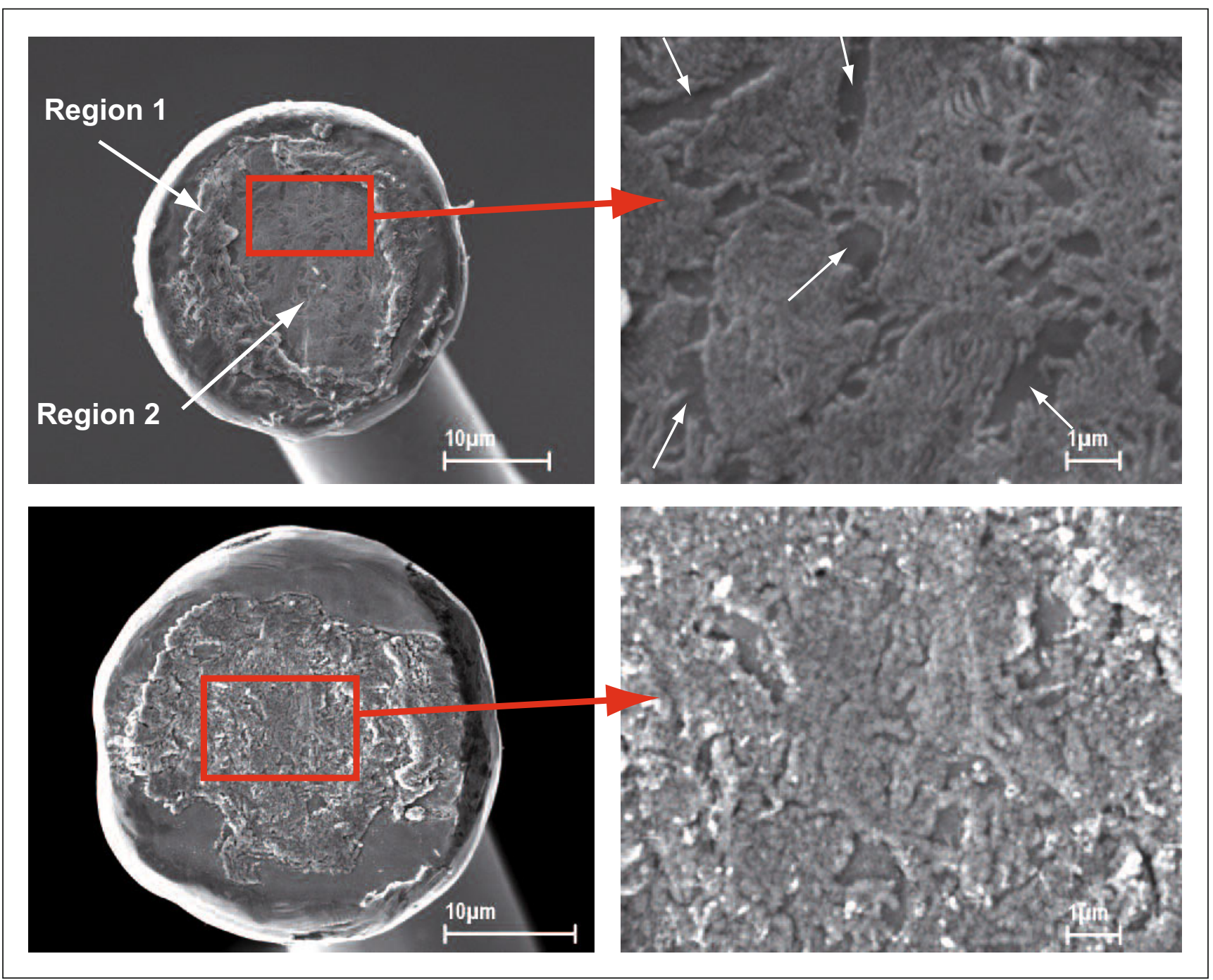

Figure 11

Example of non-uniform coverage obtained with the same wire, device and machine, bonded at different geographical locations. (a) average coverage of $68 \pm 4 \%$. Intermetallics form a thick outer ring and a thin string-like pattern at the centre (b) $62 \pm 4 \%$ 


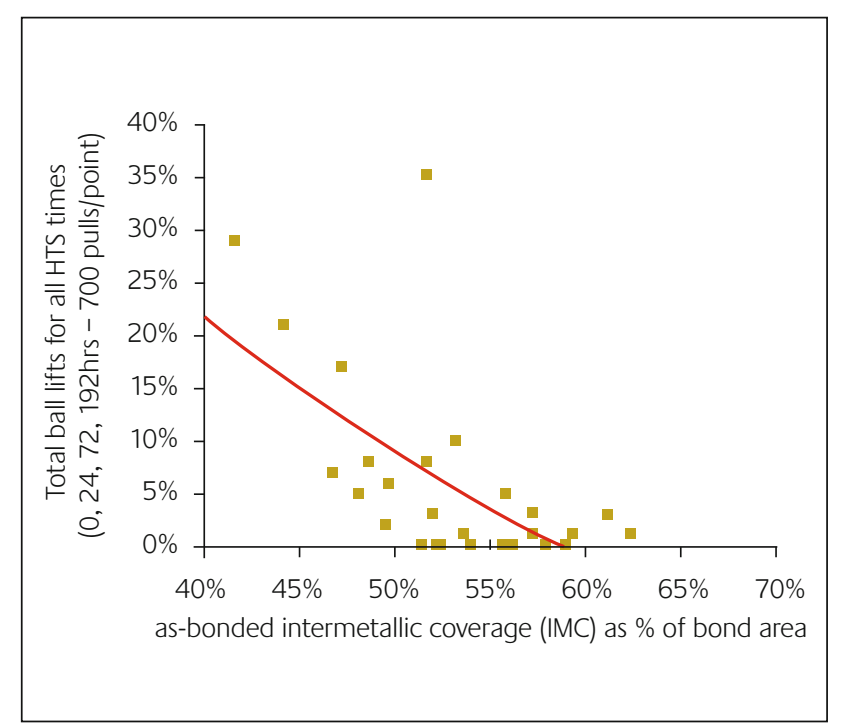

\section{Figure 12}

Example of the effects of coverage on ball-lifts for gold ballbonds on Al metallisation aged at $175^{\circ} \mathrm{C}$ [7]. Line is to guide the eye only and does not imply any data fitting

\subsection{Coverage morphology}

Simply measuring intermetallic coverage and obtaining a high value does not give a complete understanding of the mechanical robustness of a ballbond. Very often intermetallic coverage can be high and yet during reliability tests, voids and cracking may develop that ultimately lead to ball lifts. In general, high intermetallic coverage is not a guarantee of mechanical robustness but high coverage is often correlated with reduced frequencies of ball lifts for example [7].

The amount of IMC and the IMC morphology are both important in assessing the quality of the bonding process. The morphology depends upon how the ball slides against the bondpad, which can affect the way in which Au and Al intermix. Variations in coverage density, shape, thickness and the existence of unbonded areas are related to the manner in which the various parts of the ball are in contact with and slide against the Al bondpad, which also depends on the ultrasonic amplitude (USC or current in machine units), the friction coefficient between the $\mathrm{Au}$ and $\mathrm{Al}$ and the bond force. The morphology can be used to understand how well ultrasonic energy is transmitted to the interface. Very uneven $\mathrm{IMC}$, even if high, could be related to non-optimum bonding parameters. SEM is the only option for studying coverage morphology. Optical microscopy simply does not have the required resolution or magnification. Ideally, uniform and even coverage morphology should be aimed for but in some cases, coverage appears concentrated or thicker at certain parts of the ball-pad interface. An example of very even morphology is shown in Fig. 10(a). The IMC is high and has uniform depth and appearance across the whole ball. An example of non-uniform coverage is shown in Fig. 11 for the same devices bonded in different geographical locations. In Fig 11(a), the coverage is high but is significantly thicker at the outer region of the ball, suggesting that mechanical contact and friction is higher at that region than at the centre of the ball. The presence of non-uniform coverage does not guarantee failure in reliability tests such isothermal ageing tests (commonly referred to as high temperature storage and abbreviated to HTS), but the uneven coverage could affect local weld strength and diffusion. In the event of a failure in reliability, the packaging engineer or failure analyst would probably have doubts about the robustness of the process that could make root cause analysis more difficult. The presence of more uniform IMC could help the failure analyst eliminate bonding process parameters as a major influence, or at least help in prioritising them during root cause analysis.

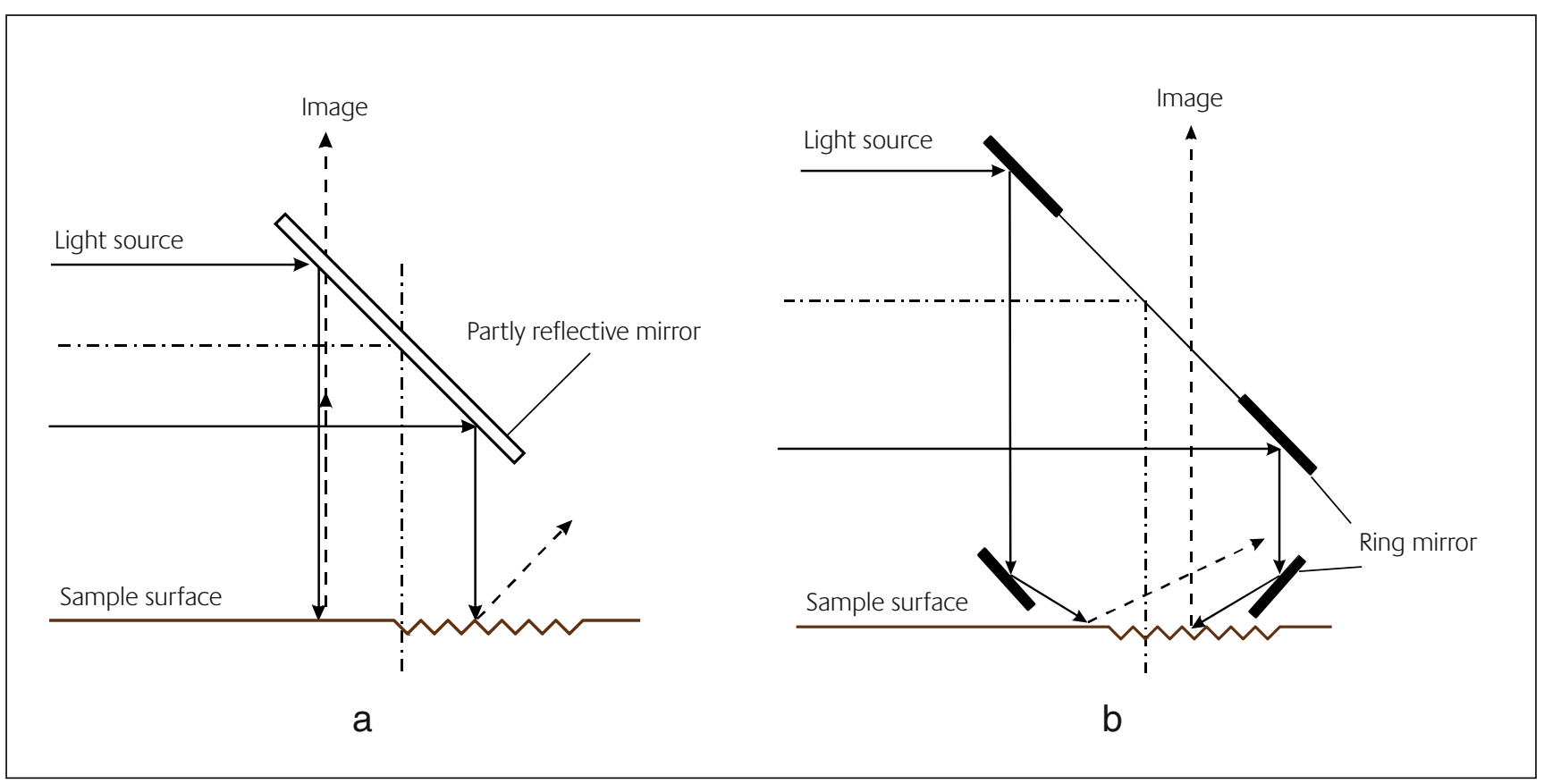

Figure 13

Schematic view of the beam path in (a) bright field (BF) (b) dark field (DF) 
Table 2

Comparison of Light and Scanning Electron Microscopies for Intermetallic Coverage Identification and Measurement

\section{Objective}

Differentiation of contamination from true physical features

Ease of use and speed
Light microscopy

Difficult to distinguish between intermetallic and contamination due to rough surfaces and reflection

If samples are prepared correctly, faster to get overview of coverage
SEM

Easier to resolve features that distinguish between intermetallic and contamination

Slower because it needs vacuum

Slower because it needs vacuum

(pump down time) and coating

if devices are not conductive
Identification of true contact area
Can be confusing due to reflection

if sample surface is not flat.

Dark field can help.

Resolution is lower than SEM
True contact area can be more easily distinguished by physical features due to higher resolution

\subsection{Recommendations for the amount of coverage}

There is no rigorous science behind the relationship between intermetallic coverage and reliability but high coverage can reduce ball lifts, as shown in Fig. 12 [7]. Generally, high coverage equals a stronger interface and the greater the bonded area, the less will be the effect of losing some of that area during intermetallic growth. Recommendations for coverage in gold ballbonds are shown in Table 1, and are derived purely from experience. However, coverage measurement without an understanding of morphology is insufficient to assess the reliability as discussed above, and the coverage measurement alone does not factor in the effects of ball deformation, distribution of residual stress and bonding process parameters. There are clearly interdependencies between process parameters that can affect both the amount of coverage and morphology.

\section{Conclusions}

SEM is the preferred method of imaging and measuring IMC in the early stages of package assembly qualification. However, in mass production, optical imaging is preferred because it requires less set-up. However, during the transition from qualification into mass production, it is important to correlate SEM and optical measurement of coverage and quantify the relationships between coverage measured by the two methods. Correct training in the use of both SEM and light microscopy is important, particularly in the use of lighting and the correct lenses in the latter technique as well as the detrimental effect that surface contamination and sample misalignment can have on IMC measurement. For quick reference, the main points are summarised in Table 2.

Coverage morphology however can only be assessed using SEM and may be important in understanding the differences between poor and good performing wires in relation to bonding process parameters and reliability.

\section{About the authors}

Mr Frank Wulff is Manager for Materials Development \& Characterization at Kulicke \& Soffa's bonding wire R\&D facility in Singapore and has been with K\&S for 10 years. Prior to joining Kulicke \& Soffa, Mr Wulff worked in industrial failure analysis and materials characterization for CEM Research Institute (Now Fraunhofer) for 6 years. His research interests include materials design, characterization, materials processing and reliability.

Dr Christopher Breach is currently Clobal Director of Applications and Field Service Operations for RVSI LLC, a leading US based manufacturer of automated optical imaging systems used in 2-D and 3-D inspection of microelectronics packages and wafers in the semiconductor industry. He is currently based in Singapore. Prior to joining RVSI he was Director of R\&D and Product Development for Kulicke \& Soffa's bonding wire R\&D facility in Singapore for $41 / 2$ years and in the previous 9 years held university and industry based positions in the areas of physics of interfaces, contact and fracture mechanics, manufacturing and materials process science and technology in Singapore and the United Kingdom. Dr Breach's current technical and research interests are focused on the science and industrial applications of optical inspection and metrology. He remains actively engaged in studies of solid state chemistry, physics and metallurgy of intermetallic compounds, inorganic alloys, microalloys and polymer physical chemistry.

\section{References}

1 M. Mayer, J. Schwizer, Proc. Int. Symp. On Microelectronics (IMAPS), Denver 2002 p626

2 M. Mayer, O. Paul, D. Bolliger, H. Baltes, IEEE CPMT 23(2) (2000) 393

3 J. Schwizer, M. Mayer, O. Brand, Force Sensors for Microelectronics Packaging. Springer Series in Microtechnology and MEMS 2005

4 C.D. Breach, F. Wulff, Microelectronics Reliability 44 (2004) 973

5 D.K. Schroder, Ch. 9 of Semiconductor Device Characterization, Wiley Interscience 1998

6 F. Wulff, T Ahrens, Struers Journal of Materialography 32 (1998) 9

$7 \quad$ L. Levine, unpublished 


\section{Appendix: Basic optical microscopy}

Light microscopes are widely used for intermetallic phase coverage analysis. The Rayleigh theoretical resolution of a light microscope is defined by $[5,6]$

$$
s=0.61 \frac{\lambda}{n \sin \theta}=0.61 \frac{\lambda}{N A}
$$

Where $\mathrm{s}$ is the distance between two points or lines visible separated from each other (higher resolution corresponds to smaller $s$ ), $\lambda$ is the wavelength of the light. $n$ is the refractive index of the medium separating the object from the objective, $\theta$ is the half-angle of the objective lens. NA is the numerical aperture of the lens, which is usually engraved on the lens and expresses the resolving power of the lens and the brightness of the image it forms. The higher $N A$, the higher the lens quality. High resolution corresponds to small $s$ and as high a value of $N A$ as possible. However, high NA corresponds to small depth of field (the thickness of the object that is simultaneously in focus) and small working distance (WD, the distance from the focus point of the object plane and the front surface of the objective). The depth of focus is given by

$$
D_{\text {focus }}=\frac{\lambda}{n N A^{2}}
$$

and the depth of field is given by

$$
D_{\text {field }}=\frac{\sqrt{n^{2}-N A^{2}}}{4 N A^{2}} \lambda=\frac{\sqrt{\left(n / N A^{2}-1\right.}}{N A} \lambda
$$

$D_{\text {focus }}$ and $D_{\text {field }}$ decrease with smaller $N A$ but resolution increases. Resolution can be increased by making $s$ smaller, which can be achieved by changing the light source with filters. Normal objectives may have WDs of $3 \mathrm{~mm}$ (20X magnification) while long WD objectives may have $11 \mathrm{~mm}$ WD (20X magnification, 0.4NA). A lens with NA of 0.55, gives a theoretical resolution $s$ of $521 \mathrm{~nm}$ when using blue light (wavelength $470 \mathrm{~nm}$ ), while a lens with high NA of 0.95 has a theoretical resolution of $302 \mathrm{~nm}$.

Optical imaging is primarily used for examination of planar surfaces. The two most fundamental operating modes of a light microscope are Bright Field (BF) and Dark Field (DF). The principle of BF mode is illustrated in Fig. 13(a), which shows how light reflected from a flat surface is sent back to the objective lens via a partially reflective mirror to form an image. Sample illumination is facilitated by a light source directed from the side that is reflected by the mirror onto the sample. Surface features that scatter light away from the objective lens appear dark while surface features that reflect light back to the objective lens appear brighter.
The DF field illumination mode uses a light source that sent through a ring mirror into the optical path of the microscope to the objective lens as illustrated in Fig. 13(b). Using such an arrangement the specimen surface is obliquely illuminated and instead of the light from the rough surfaces being scattered away from the objective, it is scattered towards the objective. Thus, contrary to bright field imaging, plane surfaces appear dark, rough areas, steps and edges appear bright. DF microscopy is particularly useful for highlighting small surface irregularities that are difficult to see with BF microscopy. 